\title{
Annulus of Collagen Fibrils in Mouse Cornea and Structural Matrix Alterations in a Murine-Specific Keratopathy
}

\author{
Andrew J. Quantock, ${ }^{1}$ Sally Dennis, ${ }^{1}$ Wakako Adachi, ${ }^{2}$ Shigeru Kinoshita, ${ }^{2}$ Craig Boote, ${ }^{1}$ \\ Keith M. Meek, ${ }^{1}$ Yoshibumi Matsushima, ${ }^{3}$ and Masayoshi Tachibana ${ }^{3}$
}

Purpose. Mouse corneas were investigated to see whether a limbal annulus of corneal collagen exists as in humans. Mice with corneas predisposed to topographical changes (the SKC strain) were also examined, to establish the size and spacing of stromal collagen fibrils and the integrity of the annulus.

Methods. X-ray diffraction was used to measure collagen fibril spacing and diameter in normal (the BALB/c strain; four male, two female) and SKC (six male and six female) corneas and to identify the degree of preferred collagen orientation at $200-\mu \mathrm{m}$ intervals across two BALB/c and four SKC corneas.

Results. The average collagen fibril diameter measured 35.5 $\mathrm{nm}$ in 3-month-old BALB/c corneas, and $36.9 \mathrm{~nm}$ and $37.0 \mathrm{~nm}$, respectively, in corneas of age-matched male and female SKC mice. In male and female SKC corneas, average collagen interfibrillar Bragg spacing was significantly higher (64.5 and 59.9 $\mathrm{nm}$, respectively) than in corneas of BALB/c mice $(49.7 \mathrm{~nm})$. Circumferentially aligned collagen, indicative of a limbal annulus of fibrillar collagen $2.2 \mathrm{~mm}$ in diameter, was identified in mouse cornea. On occasion, this was disturbed in the SKC phenotype.

Conclusions. Collagen fibrils are marginally larger in the corneas of SKC mice than in the corneas of BALB/c mice and are considerably more widely spaced. An annulus of fibrillar collagen probably exists near the limbus of the normal mouse cornea that may help promote biomechanical stability and maintain corneal shape. A loss of structural integrity in the annulus of some SKC mice may predispose the corneas to biomechanical instability and shape changes. (Invest Ophthalmol Vis Sci. 2003;44:1906-1911) DOI:10.1167/iovs.02-0884

$\mathrm{T}$ he corneal stroma is a tough, transparent connective tissue composed of uniformly thin collagen fibrils, regularly spaced and stacked in a lamellar array. ${ }^{1}$ It has a characteristic

From the ${ }^{1}$ Structural Biophysics Group, Department of Optometry and Vision Sciences, Cardiff University, Cardiff, Wales, United Kingdom; the ${ }^{2}$ Department of Ophthalmology, Kyoto Prefectural University of Medicine, Kyoto, Japan; and the ${ }^{3}$ Saitama Cancer Center, Saitama, Japan.

Supported by a Medical Research Council Programme grant, a Biotechnology and Biological Sciences Research Council Direct Access Beamtime award, and grants-in-aid from the Ministry of Education, Science, Sports, and Culture of Japan and the Japan Society for the Promotion of Science.

Submitted for publication August 29, 2002; revised November 14, 2002; accepted December 5, 2002.

Disclosure: A.J. Quantock, None; S. Dennis, None; W. Adachi, None; S. Kinoshita, None; C. Boote, None; K.M. Meek, None; Y. Matsushima, None; M. Tachibana, None

The publication costs of this article were defrayed in part by page charge payment. This article must therefore be marked "advertisement" in accordance with 18 U.S.C. $\$ 1734$ solely to indicate this fact.

Corresponding author: Andrew J. Quantock, Biophysics Group, Department of Optometry and Vision Sciences, Cardiff University, Redwood Building, King Edward VII Ave., Cathays Park, Cardiff CF10 3NB, UK; quantockaj@cf.ac.uk. fine structure that is believed to be crucial if the cornea is to transmit and help focus light. This is because light transmission requires some sort of order in the lateral packing of the collagen fibrils. ${ }^{2-4}$ Moreover, because corneal shape helps determine refractive status and given that collagen fibrils are stronger along their axes, a compelling argument can be made in support of a role for collagen fibrillar arrangement and orientation in the promotion of biomechanical stability and, as a consequence, the maintenance of corneal shape. With this in mind we note that within an individual lamella, collagen fibrils lie more or less parallel to one another in the plane of the cornea, whereas angles subtended between fibrils in adjacent lamellae tend to be fairly large. ${ }^{1}$ It is perhaps relevant, then, that Daxer and Fratzl ${ }^{5}$ have documented profound alterations in the fibrillar organization in the central 7- $\mathrm{mm}$ zone in human corneas with keratoconus and concluded that they may contribute to biomechanical instability and shape changes.

We suspect that collagen orientations in more peripheral regions may also be instrumental in maintaining the proper shape of the cornea, and a region of particular interest is the limbus-the corneoscleral junction-where tissues with different radii of curvatures meet and where the circumferential tension is at least twice as high as in neighboring regions. ${ }^{6}$ Here, in humans at least, proportionally more collagen fibrils than elsewhere in the cornea run circumferentially, and in doing so form a circumcorneal annulus of fibrillar collagen that is believed to be important for proper corneal curvature..$^{7-9}$

To discover whether an annulus of fibrillar collagen exists near the limbus of the mouse cornea as it does in humans, we initiated a series of $\mathrm{x}$-ray-scattering experiments using synchrotron radiation (see Meek and Quantock ${ }^{10}$ for a review). These included an investigation of structural changes in the corneas of SKC mice, a recently reported strain in which the males are prone to the development of misshapen corneas. ${ }^{11}$ To this end, a series of $\mathrm{x}$-ray fiber diffraction patterns from normal (the BALB/c strain) and SKC corneas were obtained and analyzed to determine collagen interfibrillar spacing and fibril diameter, as well as the spatial orientation of collagen molecules and fibrils in the plane of the cornea as a function of position across the cornea. Data are highly illustrative, because they represent an average throughout the whole region of the cornea through which the x-ray beam passes, and structural information of this type has usually been acquired from mouse corneas in studies of species differences ${ }^{12,13}$ and transgenic defects. ${ }^{14}$ The present work provides the first evidence of a circumcorneal annulus of fibrillar collagen near the limbus in the mouse cornea and indicates occasional alterations in the stromal ultrastructure in SKC mice.

\section{Materials ANd Methods}

\section{Specimens}

Twelve corneas were obtained from 3-month-old SKC mice (three male, three female), a strain derived from BALB/c, CF\#1, and STS in 
which the males are predisposed to spontaneous development of conical corneas. ${ }^{11} \mathrm{Six}$, age-matched BALB/c corneas (four male, two female) were similarly collected. Mice were killed with an overdose of intraperitoneal pentobarbital, and, at all times, the ARVO Statement for the Use of Animals in Ophthalmic and Vision Research was adhered to, as were local rules. Corneas, typically measuring $3 \mathrm{~mm}$ in diameter, were carefully excised by one of the authors (WA). Immediately after excision, specimens were wrapped in clear plastic wrap and placed in a freezer at $-80^{\circ} \mathrm{C}$. A few days later, they were shipped via express carrier in dry ice to Cardiff and stored at $-80^{\circ} \mathrm{C}$ before $\mathrm{x}$-ray-scattering experiments at the synchrotron, a large-particle accelerator that generates intense electromagnetic radiation. Previous work has shown that the x-ray diffraction patterns and the data obtained from them are unchanged after freezing and thawing. ${ }^{15}$

\section{X-ray Data Collection}

All the x-ray diffraction work was performed at the Synchrotron Radiation Source (SRS; Daresbury Laboratory, Cheshire, UK). Low-angle $\mathrm{x}$-ray-scattering patterns (for fibril diameter and interfibrillar spacing measurements) were collected on station 2.1; high-angle patterns (for orientation measurements) on station 14.1. More details of the approach can be found in a recent review. ${ }^{10}$

\section{Low-Angle X-ray Diffraction}

All BALB/C and SKC corneas were individually mounted in a specimen chamber between two sheets of mylar and allowed to thaw as they were positioned in the path of a monochromatic $\mathrm{x}$-ray beam $(\lambda=$ $0.154 \mathrm{~nm}$ ) focused to $1 \times 0.5 \mathrm{~mm}$ (horizontal $\times$ vertical) at the specimen. Low-angle $x$-ray diffraction patterns were recorded by opening the shutters for 2 minutes so that the $x$-rays passed through the center of each specimen. After passing through the tissue, the main beam was blocked by a lead "backstop," and the scattered x-rays recorded on a multiwire, gas-proportional area detector situated directly behind the cornea, $6.25 \mathrm{~m}$ away. An evacuated tube with mylar windows occupied the space between the specimen and the detector to minimize air scatter.

\section{High-Angle X-ray Diffraction}

For this portion of the study, six corneas (one male and one female $\mathrm{BALB} / \mathrm{c}$ and two male two female SKC) were investigated. As for the low-angle work, corneas were individually mounted between mylar in the path of a monochromatic $x$-ray beam, this time with a wavelength of $0.1488 \mathrm{~nm}$, and focused so that it measured $200 \times 200 \mu \mathrm{m}$ (vertically $\times$ horizontally) at the specimen. Again, the straight-through beam was stopped by a lead backstop, and this time wide-angle diffraction patterns were recorded on a CCD x-ray detector located $20 \mathrm{~cm}$ from the specimen. All exposures lasted 3 minutes. To obtain collagen orientation data as a function of position across the cornea, we generated a series of $x$-ray patterns across a complete vertical meridian of the specimen. (Here, the tissue is mounted in the x-ray beam in no particular orientation, and therefore the vertical-horizontal terms used herein refer to experimental details, not to anatomic meridians in situ). A dedicated translation stage interfaced with the x-ray shutter was used to move the specimen vertically between $x$-ray exposures, and, to scan the whole length of the vertical meridian, we obtained diffraction patterns at $200-\mu \mathrm{m}$ intervals with the $200-\mu \mathrm{m}$ x-ray beam from the lower edge of the corneal specimen to its upper edge.

\section{Data Analysis}

Low-Angle X-ray Diffraction. Low-angle x-ray patterns $(512 \times 512$ pixels) were analyzed with purpose-written Unix software and statistics and graphics packages (Statistica; Statsoft, Tulsa, OK; and Excel; Microsoft Corp., Redmond, WA) to obtain the average collagen fibril diameter and mean center-to-center collagen fibril Bragg spacing.

$\mathrm{X}$-ray patterns were first normalized by using readings taken by an ion chamber located between the incident $\mathrm{x}$-ray beam pipe exit and the specimen. This was intended to correct for the natural decay of $\mathrm{x}$-ray intensity and/or its increase after a scheduled synchrotron refill. To correct for any nonlinearities in the detector, each normalized image was then divided, pixel by pixel, by a detector response pattern obtained from a 420-minute exposure to a radioactive source $\left(\mathrm{Fe}^{55}\right)$. Next, a vertical scan, 48 pixels wide, of x-ray intensity versus radial position was generated across the center of each pattern to produce an intensity profile of the first-order equatorial (i.e., collagen interfibrillar) $\mathrm{x}$-ray reflection. The $\mathrm{x}$-ray intensity profile was then summed about its center and multiplied by radial position. The multiplication accounted for the fact that a linear scan had been taken across a circular $\mathrm{x}$-ray pattern, with the result that $\mathrm{x}$-ray reflections farther from the centers of the patterns were spread over greater arcs. Finally, background x-ray scatter from nonfibrillar material was subtracted. From the position of the interfibrillar reflection and after calibration of the system with meridional reflections from rat tail tendon $(D=67 \mathrm{~nm}$ ), we obtained a measure of the average center-to-center collagen fibril Bragg spacing for a $1 \times 0.5-\mathrm{mm}$ region in the center of each cornea. The first subsidiary maximum in the low-angle patterns was also analyzed, as described previously, ${ }^{10,16}$ to provide the mean diameters of the stromal collagen fibrils.

High-Angle X-ray Diffraction. As for the low-angle work, high-angle x-ray patterns (again, $512 \times 512$ pixels) were analyzed with Unix-based software followed by statistics and graphics programs (Statistica, MS Excel, and Optimas, Bothell, WA). In these patterns, x-ray reflections are generated by regularly spaced collagen molecules within fibrils. These intermolecular reflections are generally circular, and any variation in $\mathrm{x}$-ray intensity around the reflection is indicative of a preferred orientation of collagen molecules within the plane of the cornea (a matter that will be addressed in greater depth later). All intermolecular reflections were evaluated to generate a parameter that is indicative of the amount of preferentially aligned corneal collagen in the region of the cornea from which that particular x-ray pattern had been obtained.

To achieve this we first normalized the $\mathrm{x}$-ray patterns to account for beam decay, by using the measured intensity of $x$-ray scatter produced by mylar. Next, we computed the x-ray intensity at 256 points around the center of each $\mathrm{x}$-ray pattern and repeated this for 80 concentric circles on all x-ray diffraction patterns examined (circles were chosen to encompass the region of the x-ray pattern containing the collagen intermolecular reflections). Background scatter caused by flare from the backstop, as well as by $\mathrm{x}$-ray scatter from nonfibrillar material, was then removed by subtracting a power law fitted to either side of the collagen intermolecular peak on the plot of scattering intensity versus radial position. This was repeated for all 256 radial intensity points, to account for the uneven flare from the backstop and the changes in background with radial direction. Next, a $360^{\circ}$ radial integration was produced around each interfibrillar reflection, and from it a numerical orientation index was calculated defined as the amount of aligned collagen divided by the amount of total fibrillar (i.e., aligned plus isotropic) collagen, as described previously. ${ }^{17}$

\section{Results}

\section{Fibril Size and Separation}

Statistical analyses by ANOVA and separation-of-means tests revealed that the average diameter of corneal collagen fibrils in SKC mice calculated from low-angle x-ray diffraction patterns is significantly higher than the average diameter in BALB/c mice for both SKC males $(P<0.001)$ and females $(P<0.001$; Table 1). Fibril diameters in male and female SKC corneas are not significantly different $(P=0.766)$. X-ray data also indicates that the average center-to-center collagen interfibrillar Bragg spacing in 3-month-old BALB/c mice measures approximately $50 \mathrm{~nm}$ (Table 1), with no appreciable difference between males and females. This is similar to previously quoted data 
TABLE 1. Average Collagen Fibril Diameter and Interfibrillar Spacing in 3-Month-Old BALB/c and SKC Mouse Corneas

\begin{tabular}{lcc}
\hline \multicolumn{1}{c}{ Mouse Strain } & Fibril Diameter & $\begin{array}{c}\text { Interfibrillar Bragg } \\
\text { Spacing }\end{array}$ \\
\hline BALB/c $(n=6)$ & $35.5 \pm 0.3$ & $49.7 \pm 1.3$ \\
SKC male $(n=6)$ & $36.9 \pm 0.2$ & $64.5 \pm 3.8$ \\
SKC female $(n=6)$ & $37.0 \pm 0.2$ & $59.9 \pm 0.9$ \\
\hline
\end{tabular}

Data are mean nanometers \pm SE.

$(\approx 48 \mathrm{~nm})$ for 6 -month-old mice calculated in the same manner. ${ }^{14}$

The average fibril spacing quoted herein (Table 1) is for the interfibrillar Bragg spacing, a value that does not take into account the mode of packing of the stromal collagen fibrils. If, like Worthington and Inouye, ${ }^{16}$ we assume liquidlike packing of fibrillar collagen in cornea, we must multiply the Bragg spacing by a factor of 1.12 to arrive at the actual center-tocenter interfibrillar collagen spacing. In the SKC phenotype, collagen interfibrillar Bragg spacing was consistently higher than in BALB/c mice, and this was the case in females $(P=$ $0.008)$ and males $(P<0.001$; Table 1$)$. As was the case for fibril diameters, there was no difference in collagen interfibrillar spacing between male and female SKC groups $(P=0.156)$. We should point out, however, that corneas of male SKC mice exhibited a considerably higher variation in interfibrillar spacing than did corneas from female SKC and BALB/c mice, as indicated by the standard errors in the respective data sets (Table 1).

\section{Fibril Orientation}

An intermolecular x-ray reflection was identified on $x$-ray patterns from all corneas examined. This reflection arises because of interference between $\mathrm{x}$-rays scattered by collagen molecules within fibrils, and an appreciation of how it is formed allows us to elicit information about how many collagen molecules as a proportion of the total number of collagen molecules lie in a particular orientation in the plane of the cornea.

First, because the long, thin collagen molecules that make up the corneal collagen fibrils as a whole tend to lie along the fibrillar axis, we take collagen molecular alignment to reflect collagen fibrillar alignment. An intermolecular x-ray reflection from a single, isolated lamella in the corneal stroma in which all collagen fibrils run in the same direction would appear as two fairly well-defined diffraction maxima located either side of a line that runs in the same direction as the collagen fibrils. It follows that a stack of lamellae with a totally heterogeneous radial distributionthat is, constructed so that, when averaged throughout the whole depth of the cornea, no direction in the plane of the cornea contains more fibrils than any other direction-gives rise to a circular intermolecular reflection of uniform intensity around its circumference. The upshot is that corneas in which some directions possess proportionally more collagen fibrils than other directions give rise to lobed intermolecular $\mathrm{x}$-ray reflections, and these reflections, by definition, contain information about the relative overall orientation of collagen fibrils in the plane of the cornea.

It was immediately evident that intermolecular reflections obtained from $200 \times 200 \mu \mathrm{m}$ regions at the upper and lower edges of the corneal specimens from BALB/c mice were not of uniform intensity. Intermolecular reflections obtained from central regions of these same corneas, however, were (Fig. 1).

Invariably, $x$-ray reflections from the $200 \times 200 \mu \mathrm{m}$ areas just inside the upper and lower edges of the BALB/c corneas possessed two lobes at the 12 and 6 o'clock positions. This indicates that proportionally more collagen fibrils in these regions of the tissue run horizontally-an observation that is consistent with the existence of a circumferential annulus of collagen near the limbus, given that the tangential components of a vertical circle at its top and bottom edges are horizontal.

To quantify the level of fibrillar orientation indicated by each x-ray pattern, we generated a circular integration of $\mathrm{x}$-ray intensity around each $360^{\circ}$ intermolecular reflection. Clearly, a simple, flat trace with no peaks above the noise threshold would be expected by the integration around a perfectly uniform intermolecular reflection, but if regions of elevated $\mathrm{x}$-ray intensity existed in the intermolecular reflection, they would be denoted by peaks in the circular integration trace. All circular integrations generated from $\mathrm{x}$-ray patterns taken near the edges of the specimens contained two peaks. In contrast, high-angle diffraction patterns obtained from more central regions possessed intermolecular reflections that appeared uniformly homogeneous on visual inspection, and the circular integrations confirmed this fact. Representative traces are shown in Figure 2 and are consistent with the presence of circumferential collagen near the corneal limbus in the eyes of $\mathrm{BALB} / \mathrm{c}$ mice.

To discover by how much intermolecular reflections from different $200 \times 200 \mu \mathrm{m}$ regions of a cornea were lobed, we calculated a numerical index of intensity, by dividing the area under the peaks in the circular integrations but above homogeneous x-ray scatter by the total area under the peaks, including homogeneous scatter (Fig. 2). In effect, we divided x-ray
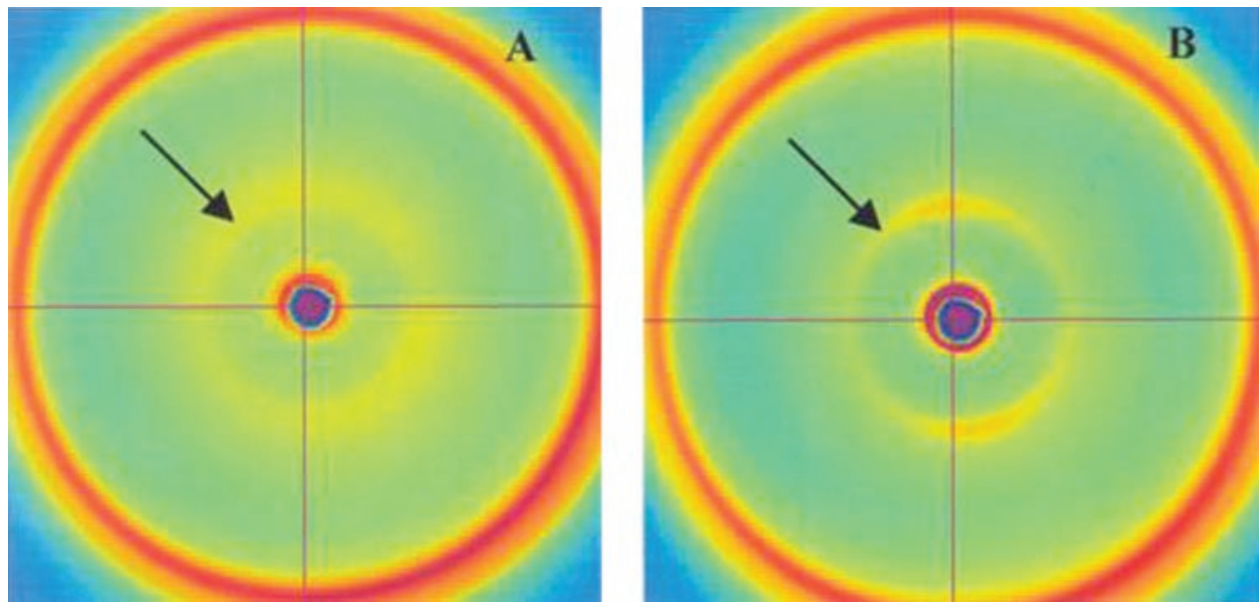

FIGURE 1. High-angle synchrotron $x$-ray diffraction patterns from $200 \times$ $200 \mu \mathrm{m}$ regions near the center (A) and edge (B) of a 3-month-old $\mathrm{BALB} / \mathrm{c}$ cornea. The central circle is the shadow of a lead backstop used to prevent the main $\mathrm{x}$-ray beam from hitting the $x$-ray detector. Outside this, regions where scattered $\mathrm{x}$-rays were more intense are indicated in yellow-orange. Arrows: intermolecular reflections. The reflection from the center of the cornea (A) was fairly homogeneous circularly, whereas that from the edge of the cornea (B) displayed twofold rotational symmetry. 


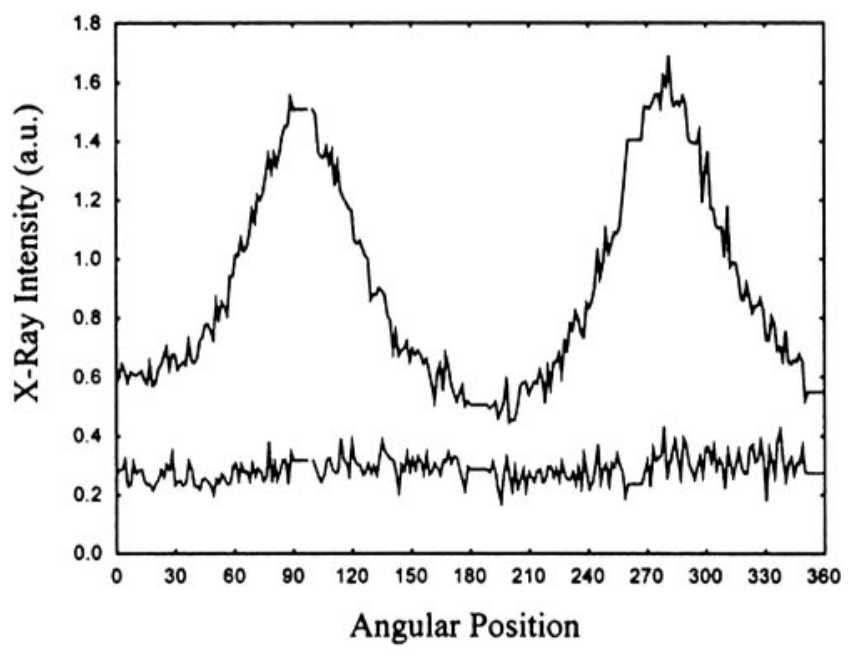

FiguRE 2. Circular $360^{\circ}$ integrations around the intermolecular reflections shown in Fig. 1, taken from the 3 o'clock position in a counterclockwise direction. Bottom trace: reflection from the central cornea (Fig. 1A); top trace: reflection from the edge of the cornea (Fig 1B). The nonuniformity in Fig 1B is clearly recognizable.

scatter from preferentially aligned collagen molecules by the $\mathrm{x}$-ray scatter from all fibrillar collagen molecules. As a result, the more lobed an intermolecular x-ray reflection, the higher its numerical index, and the higher the index, the higher the proportion of preferentially aligned collagen fibrils in that particular region of that particular cornea. Our finding of higher orientation indices at the upper and lower edges of two $\mathrm{BALB} / \mathrm{c}$ corneas is consistent with the existence of an annulus of circumferentially aligned collagen (Fig. 3). Furthermore, orientation intensity maxima lay eleven $200-\mu \mathrm{m}$ steps apart, supportive of a corneoscleral annulus in the mouse cornea 2.2 $\mathrm{mm}$ in diameter. Equivalent information from two male (Fig. 4A) and two female (Fig. 4B) SKC corneas points to a break-

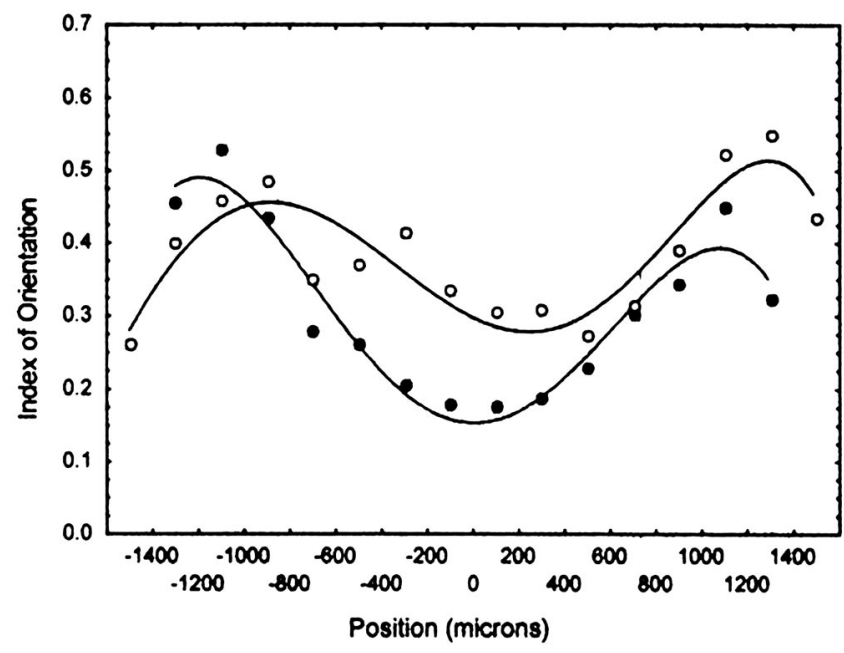

FIGURE 3. Numerical indices reflecting the extent of collagen alignment in 200- $\mu \mathrm{m}$ steps across the corneas of 3-month-old male (O) and female $(O)$ BALB/c mice. The index was higher near the edge of the cornea, indicative of proportionally more circumferentially aligned collagen and consistent with the existence of a circumcorneal annulus of collagen. That the traces are not in register simply reflects the fact that individual corneas were dissected with slightly more or less peripheral tissue intact and that $\mathrm{x}$-ray images were taken until the edge of the specimen was reached (i.e., until no x-ray pattern was recorded because the specimen was missed altogether).
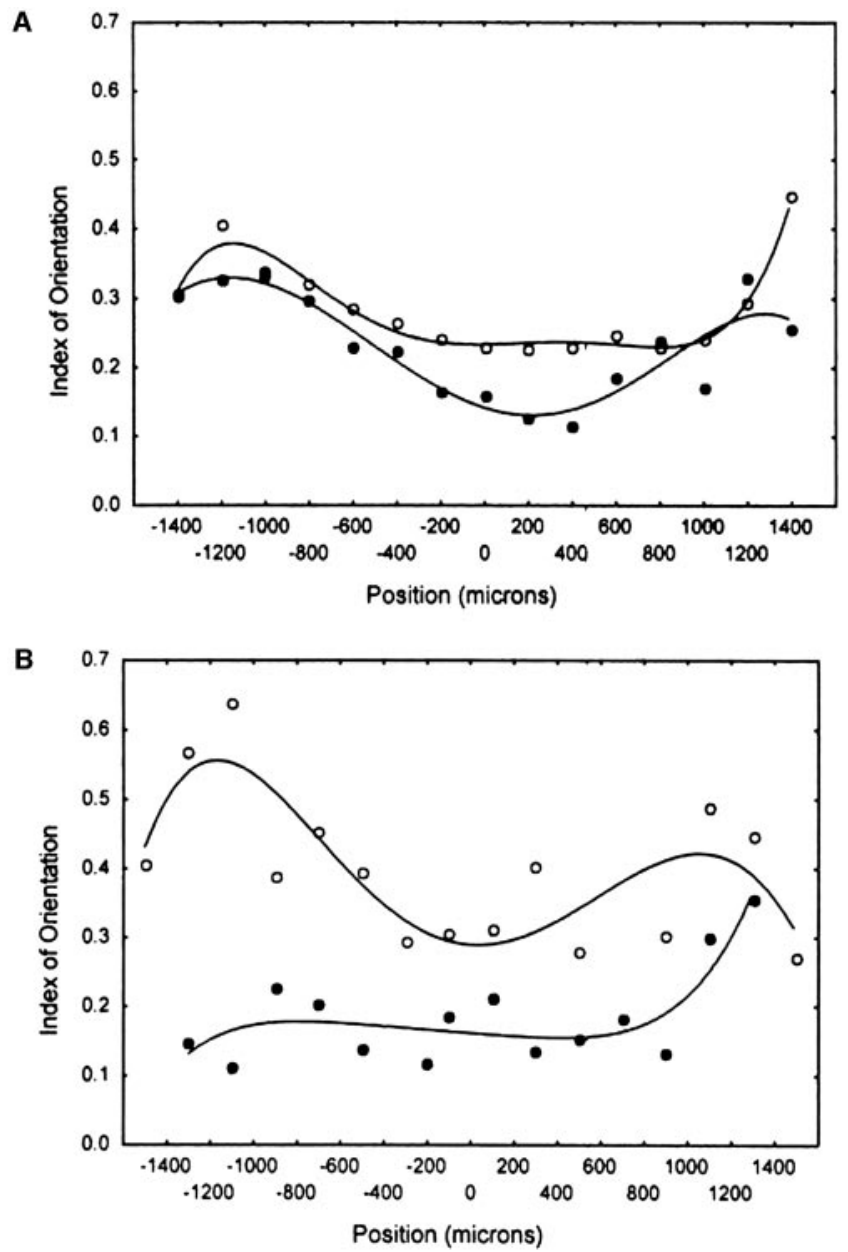

FiguRE 4. Numerical indices reflecting the extent of collagen alignment in $200-\mu \mathrm{m}$ steps across the corneas of two 3-month-old female (A) and two 3-month-old male (B) SKC mice. The tendency in normal cornea (see Fig. 3) for the index to be higher just inside the edge of the specimen seemed to break down in at least three of the four animals investigated, suggesting a possible alteration in the structural integrity of the circumcorneal annulus of collagen.

down in this arrangement in three of the four animals investigated.

\section{Discussion}

X-ray-scattering studies in the late 1980s uncovered evidence of a preferential organization of collagen fibrils in central portions of the human cornea wherein proportionally more collagen fibrils lay in the superior-inferior and medial-lateral meridians than in other meridians. ${ }^{18}$ This preferential alignment was later confirmed and quantified. ${ }^{19}$ Current $\mathrm{x}$-ray images (e.g., Fig. 1A) indicate that the central mouse cornea, like the corneas of most other species we have studied over the years, but unlike human cornea, does not contain a significant amount of collagen preferentially aligned in an orthogonal manner. Our data point to a commonality between the structures of human and mouse corneas, however, in the more peripheral regions. Recently, a circumcorneal annulus of collagen fibrils was discovered near the limbus in the human cornea in which proportionally more collagen fibrils than elsewhere run circumferentially. ${ }^{7-9}$ Because collagen fibrils are stronger along their axes, a situation can be readily envisaged in which the biomechanical stability of the cornea is contin- 
gent on the orientation of the fibrils within the plane of the cornea, and it is thought that the fibrillar annulus may well be responsible for the elevated circumferential tension at the limbus. ${ }^{6}$ The present data identified a preponderance of circumferentially aligned collagen fibrils at two opposing edges of isolated, BALB/c mouse corneas (Figs. 1, 3). Such a finding is supportive of the concept of a peripheral circumcorneal annulus of fibrillar collagen in mice, $2.2 \mathrm{~mm}$ in diameter.

Recently, an inbred line of mice was established (the SKC strain) in which conical corneas tend to develop in adult males. Initially, parallels were drawn with the human condition keratoconus, ${ }^{11}$ but the SKC keratopathy is now thought to represent a distinct form of murine corneal ectasia. Reasons for this include the fact that average collagen fibril diameter in the SKC corneas is marginally, but significantly, larger than in BALB/c corneas (Table 1), whereas previous corneal $\mathrm{x}$-ray diffraction experiments by Fullwood et al. ${ }^{20}$ found that the average collagen fibril diameter in the corneas of humans with keratoconus was essentially unchanged. They also discovered that in human corneas with keratoconus the average collagen interfibrillar spacing was no different from normal and went on to conclude that any corneal thinning that might be seen in keratoconus should not be attributed to the close packing of the population of collagen fibrils as a whole. Our current measurements indicate that the average collagen interfibrillar Bragg spacing in SKC males measured $64.5 \mathrm{~nm}$, whereas in SKC females it averaged $59.9 \mathrm{~nm}$. Both spacings are significantly wider $(P<$ 0.001 and $P=0.008$, respectively) than the $49.7 \mathrm{~nm}$ recorded in the corneas of BALB/c mice of the same age.

It might be reasonably expected that, unless there is a reduction in the amount of fibrillar collagen present, SKC corneas with wider interfibrillar spacing would be unusually thick. In fact, previous studies of the female SKC mouse ${ }^{11}$ indicate that corneas are invariably thinner than normal. Such observations are indirectly supportive of a reduction in the amount of fibrillar collagen at the centers of female SKC corneas. In SKC males, too, corneas are generally thin, and a reduction in the amount of fibrillar collagen in the center of the cornea can be envisaged. Some male SKC corneas, in contrast, are thicker than normal because of marked stromal edema. ${ }^{11}$ With this in mind, we note the high variability in the male SKC interfibrillar measurements ( $\mathrm{SE} \pm 3.8 \mathrm{~nm}$ ), and point out that one individual cornea had a remarkably high average interfibrillar spacing of $82.5 \mathrm{~nm}$. Presumably, this particular cornea was edematous, but additional experiments that directly relate corneal thickness to interfibrillar spacing are required to resolve this matter. The initial report of the SKC strain ${ }^{10}$ highlighted similarities with keratoconus in humans, but mentioned that there were distinctions-not least, the likelihood that inflammatory changes may be at play in the SKC mouse cornea. The present work indicates that from a structural viewpoint, too, the SKC strain should not act as a direct animal model of keratoconus in humans. Rather, it and similar strains, such as the JKC mouse ${ }^{21}$ hold promise for further studies of the supposed dependence of corneal shape on the collagenous architecture of the corneal stroma.

The ultrastructural changes in SKC mice compared with $\mathrm{BALB} / \mathrm{c}$ mice are clear, but represent something of a dilemma if we are trying to hold alterations in collagen packing responsible for corneal shape changes. This is simply because previous work has shown that the corneas of female SKC mice are less likely to become misshapen than are the corneas of male SKC mice, ${ }^{11}$ but the current findings point to ultrastructural alterations in the stromal matrix in both male and female SKC corneas that are of similar magnitudes. Indeed, neither the collagen diameter measurements nor the interfibrillar measurements are significantly different between male and female SKC groups $(P=0.766$ and $P=0.156$, respectively). Taken to- gether, these results seem to suggest that any predisposition of SKC males over SKC females toward development of a misshapen cornea is probably not a direct result of more widely spaced collagen fibrils that are marginally larger than normal.

The idea that collagen fibrillar orientation in the cornea might impinge on tissue stability and shape was the basis for the investigations of Daxer and Fratzl, ${ }^{5}$ who discovered that in human keratoconus buttons obtained after surgery the typical inferior-superior/medial-lateral arrangement of corneal collagen in the central cornea ${ }^{18}$ breaks down. They reasonably concluded that structural alterations may lead to tissue instability and the formation of a misshapen cornea. Of course, there are important structural differences between the corneas of mice and humans-not least, size and the considerable lamellar interweaving in the anterior stroma in humans, and it is unlikely that the shape mediation of the cornea is borne solely by the limbal annulus. Nevertheless, we believe that current evidence for the likely presence in normal murine cornea of a circumcorneal annulus of fibrillar collagen, $2.2 \mathrm{~mm}$ in diameter, identifies the mouse as a legitimate animal model for future investigations into these issues.

The present work revealed no clear, quantitative differences in the alignment of collagen in the central corneas of $\mathrm{BALB} / \mathrm{c}$ and SKC mice. It did, however, suggest that the structural integrity of the circumcorneal annulus may have been altered in three of the four SKC corneas investigated. Previous work $^{11}$ has indicated that male mice of the SKC strain are predisposed to corneal shape changes, but the structural changes in the corneal annulus that we report were manifest in SKC females, even though these mice do not tend toward development of misshapen corneas as often. Thus, the definitive establishment of a causal relationship between corneal shape and the integrity of the circumcorneal annulus awaits further investigation.

\section{Acknowledgments}

The authors thank Gunter Grossmann, Mike McDonald, and Rob Keyhoe for help at the SRS, Daresbury Laboratory and Morio Ueno and Chikako Mochida for assistance with tissue collection.

\section{References}

1. Komai Y, Ushiki T. The three-dimensional organization of collagen fibrils in the human cornea and sclera. Invest Ophthalmol Vis Sci. 1991;32:2244-2258.

2. Maurice DM. The structure and transparency of the cornea. J Pbysiol (London). 1957;186:263-286.

3. Benedek GB. Theory of transparency of the eye. Appl Opt. 1971; $10: 459-473$.

4. Farrell RA. Corneal transparency. In: Albert DM, Jacobiec SA, eds. Principles and Practice of Opbthalmology. Philadelphia: WB Saunders; 1994:64-81.

5. Daxer A, Fratzl P. Collagen fibril orientation in the human corneal stroma and its implications in keratoconus. Invest Opbthalmol Vis Sci. 1997;38:121-129.

6. Maurice DM. The cornea and sclera. In: Davson $\mathrm{H}$, ed. The Eye. London: Academic Press; 1969:489-600.

7. Newton RH, Meek KM. Circum-corneal annulus of collagen fibrils in the human limbus. Invest Opbthalmol Vis Sci. 1998;39:11251134 .

8. Newton RH, Meek KM. The integration of the corneal and limbal fibrils in the human eye. Biopbys J. 1998;75:2508-2512.

9. Meek KM, Newton RH. Organisation of collagen fibrils in the corneal stroma in relation to mechanical properties and surgical practice. J Refract Surg. 1999;15:695-699.

10. Meek KM, Quantock AJ. The use of x-ray scattering techniques to determine corneal ultrastructure. Prog Retinal Eye Res. 2001;20: 95-137. 
11. Tachibana M, Adachi W, Kinoshita S, et al. Androgen-dependent hereditary mouse keratoconus: linkage to an $\mathrm{MHC}$ region. Invest Ophthalmol Vis Sci. 2002;43:51-57.

12. Gyi TJ, Meek KM, Elliott GF. Collagen interfibrillar distances in corneal stroma using synchrotron X-ray diffraction: a species study. Int J Biol Macromol. 1988;10:265-269.

13. Meek KM, Leonard DW. The ultrastructure of the corneal stroma: a comparative study. Biophys J. 1993;64:273-280.

14. Quantock AJ, Meek KM, Chakravarti S. An x-ray diffraction investigation of corneal structure in lumican-deficient mice. Invest Ophthalmol Vis Sci. 2001;42:1750-1756.

15. Fullwood NJ, Meek KM. An ultrastructural, time-resolved study of freezing in the corneal stroma. J Mol Biol. 1994;236:749-758.

16. Worthington CR, Inouye H. X-ray diffraction study of the cornea. Int J Biol Macromol. 1985;7:2-8.
17. Quantock AJ, Boote C, Siegler V, Meek KM. Collagen organization in the secondary chick cornea during development. Invest $O p b$ thalmol Vis Sci. 2003;44:130-136.

18. Meek KM, Blamires T, Elliott GF, Gyi T, Nave C. The organisation of collagen fibrils in the human corneal stroma: a synchrotron X-ray diffraction study. Curr Eye Res. 1987;6:841-846.

19. Daxer A, Misof K, Grabner B, Ettl A, Fratzl P. Collagen fibrils in the human corneal stroma: structure and ageing. Invest Ophthalmol Vis Sci. 1998;39:644-648.

20. Fullwood NJ, Tuft SJ, Malik NS, Meek KM, Ridgway AEA, Harrison RJ. Synchrotron X-ray diffraction studies of keratoconus corneal stroma. Invest Ophthalmol Vis Sci. 1992;33:1734-1741.

21. Tachibana M, Okamoto M, Sakamoto M, Matsushima Y. Hereditary keratoconus-like keratopathy in Japanese wild mice mapped to mouse chromosome 13. Mamm Genome. 2002;13:692-695. 